\title{
Composition Effect on Shrinkage of Hollow Binary Alloy Nanospheres
}

\author{
A.V. Evteev" ${ }^{1, a}$, E.V. Levchenko, ${ }^{1, b}$, I.V. Belova ${ }^{1, c}$ and G.E. Murch ${ }^{1, d}$
}

${ }^{1}$ The University Centre for Mass and Thermal Transport in Engineering Materials, School of Engineering, The University of Newcastle, Callaghan, NSW 2308, Australia

aAlexander.Evteev@newcastle.edu.au, ${ }^{b} E$ Elena.Levchenko@newcastle.edu.au, Irina.Belova@newcastle.edu.au, dGraeme.Murch@newcastle.edu.au

Keywords: Diffusion, stability, vacancies, Monte Carlo simulation, binary alloy, hollow nanospheres.

\begin{abstract}
In this paper, a hollow random binary alloy nanosphere and initially homogeneous is considered under the approximation that the radial dependence of the vacancy formation free energy can be neglected. On the basis of a theoretical description and kinetic Monte Carlo simulations it is shown that the steady-state condition for the atomic components is not achievable during its shrinkage at any composition when the ratio of the tracer diffusion coefficients $D_{\mathrm{A}}^{*} / D_{\mathrm{B}}^{*}$ is not greater than two orders of magnitude. In the theoretical description, the dependence of the collapse time of the hollow random binary alloy nanosphere on the atomic fraction $x$ of the faster diffusing species at $x \leq 0.5$ can be estimated by using the geometric mean of the ratios of the atomic fluxes at self-diffusion and steady-state. At $x>0.5$ the ratio of the atomic fluxes approaches the self-diffusion ratio as $x$ increases.
\end{abstract}

\section{Introduction}

Hollow nanoparticles represent an important class of novel materials and attract special interest due to their broad range of advanced scale-dependent applications, for example, in photonic devices, drug delivery vehicles, nano-chemical reactors, robust carriers/catalysts, active material encapsulation, ionic intercalation, key components of ultra-lightweight structural materials, size-selective reactions [1-5]. Today, hollow nano-objects can be fabricated from a wide spectrum of materials ranging from single elements to ternary compounds [2]. The underlying mechanisms of the synthesis of hollow nanoparticles can be classified into template and template-free categories. At the present time, template synthesis is the most widely used method for materials chemists to prepare this class of materials [1]: nanomaterials with hollow interiors can be obtained after removal of the sacrificial templates, such as a porous matrix, solid nanowires or nanoparticles. For example, Sun et al., [3] have developed a simple and generic approach for the large-scale synthesis of hollow nanostructures made of $\mathrm{Au}, \mathrm{Pt}$ and $\mathrm{Pd}$. The key step of this process is the redox reaction between the surface of the Ag template and the solution of the appropriate salt precursor. Template-free techniques are based on self-organisation phenomena such as self-rolling, oriented attachment, Ostwald ripening, and, recently, the Kirkendall effect [1,2,4,5]. Using the Kirkendall effect at the nano-level, Yin et al. [4] demonstrated the formation of hollow nanospheres of cobalt oxide, sulphide and selenide starting with nanocrystals of cobalt in an environment of the non-metal. Thus significant progress in experimental work on synthesis of hollow nanoparticles has already been achieved. A number of valuable reviews on inorganic hollow nano-objects are already available $[1,2,6,7]$.

Nevertheless, it has been noted [8], that hollow nanospheres should be unstable in principle and, with time, they should have a tendency to shrink to a compact nanosphere. This is because the resulting reduction in surface area should be more advantageous from an energetic point of view: $\gamma\left(r_{\mathrm{i}}^{2}+r_{\mathrm{e}}^{2}\right)>\gamma r_{\mathrm{f}}^{2}$ ( $\gamma$ is the surface energy per unit area, $r_{\mathrm{i}}$ and $r_{\mathrm{e}}$ are the inner and external radii of a hollow nanosphere, $r_{\mathrm{f}}$ is the radius of a collapsed compact nanosphere). In accordance with [8] the mechanism of shrinking can be considered as a result of a vacancy flux from the inner surface to the external surface. The driving force for such a flux is the difference between the vacancy compositions $c_{\mathrm{V}}$ on the inner and external surfaces due to the difference of the chemical potential of the vacancies $\mu_{\mathrm{v}}=\Delta g_{\mathrm{f}}+k T \ln c_{\mathrm{V}}\left(\Delta g_{\mathrm{f}}\right.$ is the vacancy formation free energy) on the inner and external surfaces (the Gibbs-Thomson effect). 
In [9], it has been noted that in the case of a binary alloy nanoshell, the shrinkage process is more complicated. The vacancy flux $J_{\mathrm{V}}$ from inner to external surface, caused by the Gibbs-Thomson effect, leads to inward atomic fluxes $J_{\mathrm{A}}$ and $J_{\mathrm{B}}$ (A and $\mathrm{B}$ are the atomic components of the binary alloy) [9]. If diffusion of one species, for example A, proceeds faster, then A will segregate near the inner surface. The resulting composition gradient A will reduce the vacancy flux and hence will have a tendency to suppress the shrinking process [9]. The main result of the phenomenological analysis [9] is that the shrinkage rate of a hollow nanosphere in the case of a binary alloy will be controlled by the diffusion of the slower species according to the steady-state condition for the atomic components.

In [10], by theoretical description and kinetic Monte Carlo (KMC) simulations, it has been shown that for a hollow random binary alloy nanosphere with an equi-atomic (initially homogeneous) composition and neglecting the radial dependence of vacancy formation free energy, the steady-state condition for the atomic components is not reachable during shrinkage and its collapse time can be estimated with reasonable accuracy by using the geometric mean of the ratios of the atomic fluxes at self-diffusion and steady-state.

In this paper, a theoretical and KMC study of the composition effect on the rate of hollow random binary alloy nanospheres shrinkage induced by the vacancy diffusion is performed.

\section{Theory}

In a hollow nanosphere with internal radius $r_{\mathrm{i}}$ and external radius $r_{\mathrm{e}}$, the variation of the vacancy composition near the surfaces is given by the Gibbs-Thomson equations [10]:

$$
c_{\mathrm{V}}^{\mathrm{i}(\mathrm{e})}=\exp \left(-\Delta g_{\mathrm{f}}^{\mathrm{i}(\mathrm{e})} / k T\right) \exp \left(\kappa_{\mathrm{i}(\mathrm{e})} \beta / r_{\mathrm{i}(\mathrm{e})}\right),
$$

where $c_{\mathrm{V}}^{\mathrm{i}}$ and $c_{\mathrm{V}}^{\mathrm{e}}$ are the vacancy compositions, and $\Delta g_{\mathrm{f}}^{\mathrm{i}}$ and $\Delta g_{\mathrm{f}}^{\mathrm{e}}$ are the vacancy formation free energies near the inner and external surfaces of the hollow nanosphere, respectively; $\beta=2 \gamma \Omega / k T$ and $\Omega$ is the average atomic volume (the curvature is taken as positive $\kappa_{\mathrm{i}}=+1$ for the inner surface and negative $\kappa_{\mathrm{e}}=-1$ for the external surface). It should be noted that at equilibrium, the vacancy composition $c_{\mathrm{V}}^{0}$ with respect to a planar surface in a crystal system is given by $c_{\mathrm{V}}^{0}=\exp \left(-\Delta g_{\mathrm{f}}^{0} / k T\right)$, where $\Delta g_{\mathrm{f}}^{0}$ is the vacancy formation free energy near the planar surface. A simplified treatment of the Gibbs-Thomson effect for a hollow nanosphere (Eq. 1) (see [8]) assumes that $\Delta g_{\mathrm{f}}^{\mathrm{i}}=\Delta g_{\mathrm{f}}^{\mathrm{e}}=\Delta g_{\mathrm{f}}^{0}$ and that the vacancy formation free energy with respect to a planar surface in a crystal system does not depend on distance from the surface, so that $\Delta g_{\mathrm{f}}^{0}=\Delta g_{\mathrm{f}}^{\mathrm{eq}}\left(\Delta g_{\mathrm{f}}^{\mathrm{eq}}\right.$ is the vacancy formation free energy with respect to the planar surface in the bulk of the crystal system). In this approximation, Eq. 1 is [8]:

$$
c_{\mathrm{V}}^{\mathrm{i}(\mathrm{e})}=c_{\mathrm{V}}^{\mathrm{eq}} \exp \left(\kappa_{\mathrm{i}(\mathrm{e})} \beta / r_{\mathrm{i}(\mathrm{e})}\right)
$$

where $c_{\mathrm{V}}^{\mathrm{eq}}$ is the vacancy composition with respect to the planar surface in the bulk of the crystal system. From Eq. 2 it follows that $c_{\mathrm{V}}^{\mathrm{i}}>c_{\mathrm{V}}^{\mathrm{eq}}>c_{\mathrm{V}}^{\mathrm{e}}$, so a vacancy composition profile across the hollow nanoshell will be represented by a monotonic decreasing function and consequently a vacancy composition gradient across the hollow nanoshell will exist [8].

Let us consider the vacancy flux in the following form (for the pure element this form can be identified with Fick's First Law):

$$
\Omega J_{\mathrm{V}}=-D \partial c_{\mathrm{V}} / \partial r
$$

where $D$ can be considered as the controlling parameter of the vacancy motion within a nanoshell. In particular, for a pure element hollow nanosphere under the approximation of Eq. 2 we have $D=D_{\mathrm{v}}$, where $D_{\mathrm{v}}$ is the diffusion coefficient of the vacancies, which is related to the measurable tracer diffusion coefficient $D^{*}$ by $D^{*}=c_{\mathrm{V}} D_{\mathrm{V}} f_{0}\left(f_{0}\right.$ is the geometric correlation factor for the lattice). It is obvious that in the general case of binary and higher compounds more than one independently varying thermodynamic driving force should be considered. The parameter $D$ then cannot be 
expressed in terms of only diffusion coefficients but will require the use of terms containing driving forces. Therefore Fick's First Law is no longer appropriate. We will then call the parameter $D$ a controlling parameter. The radius $r_{\mathrm{i}}$ of the inner surface varies with time as:

$$
d r_{\mathrm{i}} / d t=-\Omega J_{\mathrm{V}}\left(r_{\mathrm{i}}\right)=D \partial c_{\mathrm{V}} /\left.\partial r\right|_{r=r_{\mathrm{i}}} .
$$

The external radius $r_{\mathrm{e}}$ changes can be found from the condition of the conservation of matter: $r_{\mathrm{f}}^{3}=r_{\mathrm{e}}^{3}-r_{\mathrm{i}}^{3}=$ const . To analyse the shrinking kinetics of a hollow nanosphere with the use of Eqs. 2 and 4 , the vacancy composition profile and the controlling parameter of the vacancy motion within the nanoshell must be determined using an appropriate method.

We assume that the process is in a quasi-steady-state for vacancy concentration:

$$
\partial c_{\mathrm{V}} / \partial t=-r^{-2} \partial\left(r^{2} \Omega J_{\mathrm{V}}\right) / \partial r \approx 0 \text {. }
$$

A linear approximation of the Gibbs-Thomson boundary conditions (Eq. 2) (with a maximum error of $5 \%$ when $r_{\mathrm{i}}>3 \beta$ ) gives the following expressions for the vacancy compositions at the internal and external surfaces: $c_{\mathrm{V}}^{\mathrm{i}(\mathrm{e})}=c_{\mathrm{V}}^{\mathrm{eq}}\left(1+\kappa_{\mathrm{i}(\mathrm{e})} \beta / r_{\mathrm{i}(\mathrm{e})}\right)$. Then, taking into account Eq. 5 and neglecting the radial dependence of $D$, Eq. 4 can be reduced to $[8,9]$ :

$$
d r_{\mathrm{i}} / d t=-c_{\mathrm{V}}^{\mathrm{eq}} D \beta r_{\mathrm{i}}^{2}\left(r_{\mathrm{e}}+r_{\mathrm{i}}\right) /\left(r_{\mathrm{e}}-r_{\mathrm{i}}\right) .
$$

In [11], an analytical solution to Eq. 6 has been found:

$$
\tau(\delta)=\ln \left[\frac{\left(1-\varepsilon_{0}\right)\left(1+\varepsilon_{0}\right)^{3}}{\left(1-\varepsilon_{0}^{3}\right)^{\frac{4}{3}}} \frac{\left(1-\varepsilon_{0}^{3} \delta^{3}\right)^{\frac{4}{3}}}{\left(1-\varepsilon_{0} \delta\right)\left(1+\varepsilon_{0} \delta\right)^{3}}\right]-2 \varepsilon_{0}\left(\frac{1-\varepsilon_{0}}{1-\varepsilon_{0}^{3}}-\delta \frac{1-\varepsilon_{0} \delta}{1-\varepsilon_{0}^{3} \delta^{3}}\right),
$$

where $\tau=6 c_{\mathrm{V}}^{\mathrm{eq}} D t \beta / r_{\mathrm{f}}^{3}, \delta=\varepsilon / \varepsilon_{0}, \varepsilon=r_{\mathrm{i}} / r_{\mathrm{e}}, \varepsilon_{0}=r_{\mathrm{i} 0} / r_{\mathrm{e} 0}, r_{\mathrm{i} 0}$ and $r_{\mathrm{e} 0}$ are the inner and the external radii of the hollow nanosphere at the initial point of time $t=0$. Since the linear approximation of the GibbsThomson boundary conditions is satisfactory only at $r_{\mathrm{i}}>3 \beta$, the lowest value of $\delta$ given by Eq. 7 cannot equal zero. Nevertheless, in [12] on the basis of the results of kinetic Monte Carlo simulation of a pure element hollow nanosphere it was shown that Eq. 7 describes shrinkage kinetics quite reasonably even for the case $r_{\mathrm{i} 0}<3 \beta$. Therefore, it is justifiable to substitute zero for $\delta$ in Eq. 7 in order to obtain a rather simple expression for the estimation of the collapse time of a hollow nanosphere:

$$
t_{\mathrm{c}}=k \operatorname{Tr}_{\mathrm{f}}^{3} \tau_{\mathrm{c}} / 12 c_{\mathrm{V}}^{\mathrm{eq}} D \gamma \Omega, \quad \tau_{\mathrm{c}} \approx \ln \left[\left(1-\varepsilon_{0}\right)\left(1+\varepsilon_{0}\right)^{3}\left(1-\varepsilon_{0}^{3}\right)^{-\frac{4}{3}}\right]-2 \varepsilon_{0}\left(1-\varepsilon_{0}\right) /\left(1-\varepsilon_{0}^{3}\right) .
$$

However, unlike the simplified treatment of the Gibbs-Thomson effect (Eq. 2), in reality we can expect a vacancy composition profile across the hollow nanoshell to be represented by a function with a minimum $\left(c_{\mathrm{V}}^{\mathrm{i}}>c_{\mathrm{V}}^{\mathrm{eq}}<c_{\mathrm{V}}^{\mathrm{e}}\right)$. This will be justified if we take into account that the vacancy formation free energy with respect to a planar surface in a crystal system depends on distance from the surface due to surface and subsurface relaxation, so that $\Delta g_{\mathrm{f}}^{0}+2 \gamma \Omega / r_{\mathrm{e}}<\Delta g_{\mathrm{f}}^{\text {eq }}$. Additionally, surface and subsurface relaxation processes can influence (in varying degrees) the relative changes of vacancy formation free energies $\Delta g_{\mathrm{f}}^{\mathrm{i}}, \Delta g_{\mathrm{f}}^{\mathrm{e}}$ and $\Delta g_{\mathrm{f}}^{0}$ because of the difference in sign and value of the curvature of the surfaces. Indeed, because of surface and subsurface relaxation processes we can suppose the largest relative decrease being of $\Delta g_{\mathrm{f}}^{\mathrm{e}}$ (negative curvature surface has the highest surface free energy) followed by the $\Delta g_{\mathrm{f}}^{0}$ and $\Delta g_{\mathrm{f}}^{\mathrm{i}}$. In this case, the hollow nanosphere should be in a state corresponding to the local minimum of the free energy. Accordingly, growing of the vacancy composition in the shell should result in an increase of the free energy of such a system, namely to an increase of that free energy part which is responsible for the volume of the nanoshell. Thus, some free energy barrier should obstruct the transition of the hollow nanosphere by the vacancy mechanism to the state corresponding to the global minimum of free energy (shrinkage to a solid nanosphere). Furthermore, the vacancy formation free energy near a 
surface in an alloy should be a function of atomic composition because of surface and subsurface segregation phenomena.

Next, we discuss shrinking kinetics by vacancy diffusion of a hollow binary alloy nanosphere with the assumption that a vacancy composition profile across the nanoshell can be represented by a monotonically decreasing function.

In [10], it has been shown that the Onsager flux equations [13,14] allow the vacancy flux in a hollow binary alloy nanosphere containing two atomic components $\mathrm{A}$ and $\mathrm{B}$ on a single lattice with $N_{\mathrm{A}}$ and $N_{\mathrm{B}}$ atoms $\left(\mathrm{A}_{x} \mathrm{~B}_{1-x}\right.$, where $x=N_{\mathrm{A}} /\left(N_{\mathrm{A}}+N_{\mathrm{B}}\right)$ is the average atomic fraction of $\left.\mathrm{A}\right)$ to be written in the form:

$$
J_{\mathrm{V}}=\frac{L_{\mathrm{AA}} L_{\mathrm{BB}}-L_{\mathrm{AB}}^{2}}{\left(c_{\mathrm{A}} L_{\mathrm{BB}}-c_{\mathrm{B}} L_{\mathrm{AB}}\right) J_{\mathrm{A}} /\left(J_{\mathrm{A}}+J_{\mathrm{B}}\right)+\left(c_{\mathrm{B}} L_{\mathrm{AA}}-c_{\mathrm{A}} L_{\mathrm{AB}}\right) J_{\mathrm{B}} /\left(J_{\mathrm{A}}+J_{\mathrm{B}}\right)} X_{\mathrm{V}},
$$

where $L_{\mathrm{AA}}, L_{\mathrm{BB}}$ and $L_{\mathrm{AB}}$ are the phenomenological coefficients; $c_{\mathrm{A}}$ and $c_{\mathrm{B}}$ are the compositions of species $\mathrm{A}$ and $\mathrm{B}$ (fractions of sites occupied by $\mathrm{A}$ and $\mathrm{B}$ atoms); $X_{\mathrm{V}}$ is the vacancy thermodynamic force. In writing Eq (9) it was supposed that the system is isothermal, and that external forces and any vacancy sinks or sources inside the nanoshell are absent. Then the vacancy thermodynamic force $X_{\mathrm{V}}$ is equal to minus the gradient of the chemical potential of vacancies. The thermodynamic force arising from the gradient of the vacancy chemical potential is:

$$
X_{\mathrm{V}}=-\partial \mu_{\mathrm{v}} / \partial r=-\left(k T / c_{\mathrm{V}}\right) \varphi_{\mathrm{V}} \partial c_{\mathrm{V}} / \partial r
$$

where $\varphi_{\mathrm{V}}=1+\left(k T / c_{\mathrm{V}}\right)^{-1}\left[\left(\partial \Delta g_{\mathrm{f}} / \partial r\right)+\left(\partial \Delta g_{\mathrm{f}} / \partial c_{\mathrm{A}}\right)\left(\partial c_{\mathrm{A}} / \partial r\right)\right]\left(\partial c_{\mathrm{V}} / \partial r\right)^{-1}$ can be denoted as the vacancy thermodynamic factor. It should arise if the vacancy formation free energy depends either on the distance from a surface due to subsurface relaxation or on atomic composition due to surface and subsurface segregation phenomena or on both. Therefore, the vacancy thermodynamic factor might be important for analysis of diffusion by vacancies inside a nano-object because in such a system the fractions of subsurface and bulk atoms are of comparable magnitudes. Substituting Eq. 10 into Eq. 9, we have:

$$
\Omega J_{\mathrm{V}}=-\frac{\Omega k T}{c_{\mathrm{V}}} \frac{L_{\mathrm{AA}} L_{\mathrm{BB}}-L_{\mathrm{AB}}^{2}}{\left(c_{\mathrm{A}} L_{\mathrm{BB}}-c_{\mathrm{B}} L_{\mathrm{AB}}\right) J_{\mathrm{A}} /\left(J_{\mathrm{A}}+J_{\mathrm{B}}\right)+\left(c_{\mathrm{B}} L_{\mathrm{AA}}-c_{\mathrm{A}} L_{\mathrm{AB}}\right) J_{\mathrm{B}} /\left(J_{\mathrm{A}}+J_{\mathrm{B}}\right)} \varphi_{\mathrm{V}} \frac{\partial c_{\mathrm{V}}}{\partial r} .
$$

Comparing Eqs. 3 and 11 the controlling parameter of the vacancy motion within a binary alloy nanoshell (or shrinking rate) can be written in the form:

$$
D=\frac{\Omega k T}{c_{\mathrm{V}}} \frac{L_{\mathrm{AA}} L_{\mathrm{BB}}-L_{\mathrm{AB}}^{2}}{\left(c_{\mathrm{A}} L_{\mathrm{BB}}-c_{\mathrm{B}} L_{\mathrm{AB}}\right) J_{\mathrm{A}} /\left(J_{\mathrm{A}}+J_{\mathrm{B}}\right)+\left(c_{\mathrm{B}} L_{\mathrm{AA}}-c_{\mathrm{A}} L_{\mathrm{AB}}\right) J_{\mathrm{B}} /\left(J_{\mathrm{A}}+J_{\mathrm{B}}\right)} \varphi_{\mathrm{V}},
$$

In Eq. 12, the atomic fluxes can be approximated by $J_{\mathrm{A}}^{*}$ and $J_{\mathrm{B}}^{*}-$ the atomic fluxes in the selfdiffusion experiment. Let us consider the following expression as the first approximation to the ratio of the atomic fluxes:

$$
J_{\mathrm{A}} / J_{\mathrm{B}}=J_{\mathrm{A}}^{*} / J_{\mathrm{B}}^{*}=\left(L_{\mathrm{AA}}+L_{\mathrm{AB}}\right) /\left(L_{\mathrm{BB}}+L_{\mathrm{AB}}\right) \text {. }
$$

Approximation Eq. 13 comes about if we assume that the atomic thermodynamic forces are equal $X_{\mathrm{A}}=X_{\mathrm{B}}[10]$. In this case, the controlling parameter of the vacancy motion within a binary alloy nanoshell should be equal to $D_{\mathrm{v}} \varphi_{\mathrm{V}}$ (see Eq. 12), where

$$
D_{\mathrm{V}}=\Omega k T c_{\mathrm{V}}^{-1}\left(c_{\mathrm{A}}+c_{\mathrm{B}}\right)^{-1}\left(L_{\mathrm{AA}}+2 L_{\mathrm{AB}}+L_{\mathrm{BB}}\right)
$$

is the diffusion coefficient of the vacancies [14]. On the other hand, the steady-state condition in a binary alloy can be used as another approximation for the ratio of the atomic fluxes [13]:

$$
J_{\mathrm{A}} / J_{\mathrm{B}}=J_{\mathrm{A}}^{(\mathrm{SS})} / J_{\mathrm{B}}^{(\mathrm{SS})}=c_{\mathrm{A}} / c_{\mathrm{B}},
$$

where $J_{\mathrm{A}}^{(\mathrm{SS})}$ and $J_{\mathrm{B}}^{(\mathrm{SS})}$ are the atomic fluxes at the steady-state. With this relation and Eq. 12 we have for the controlling parameter: 


$$
D^{(\mathrm{SS})}=\frac{\Omega k T}{c_{\mathrm{V}}} \frac{L_{\mathrm{AA}} L_{\mathrm{BB}}-L_{\mathrm{AB}}^{2}}{\left(c_{\mathrm{A}} L_{\mathrm{BB}}-c_{\mathrm{B}} L_{\mathrm{AB}}\right) c_{\mathrm{A}} /\left(c_{\mathrm{A}}+c_{\mathrm{B}}\right)+\left(c_{\mathrm{B}} L_{\mathrm{AA}}-c_{\mathrm{A}} L_{\mathrm{AB}}\right) c_{\mathrm{B}} /\left(c_{\mathrm{A}}+c_{\mathrm{B}}\right)} \varphi_{\mathrm{V}} .
$$

Thus in general, the controlling parameter of the vacancy motion within a binary alloy nanoshell should be confined to the interval:

$$
D^{(\mathrm{SS})}<D<D_{\mathrm{V}} \varphi_{\mathrm{V}} .
$$

From an experimental point of view it is desirable to express the lower and upper limits in Eq. 17 in terms of measurable tracer diffusion coefficients of the atomic components $D_{\mathrm{A}}^{*}$ and $D_{\mathrm{B}}^{*}$ of a binary alloy. However, for concentrated alloys substantial progress in developing of the relationships between the phenomenological coefficients and the tracer diffusion coefficients of the atomic components has been made only for the well-known random alloy model. For this reason, the random alloy model introduced first by Manning [13] is frequently used as a reference point for interpreting tracer and chemical diffusion data in concentrated alloys. In a binary random alloy, the atomic components $\mathrm{A}$ and $\mathrm{B}$ exchange with a vacancy with average jump frequencies $w_{\mathrm{A}}$ and $w_{\mathrm{B}}$, respectively. For a cubic crystal the relations between $w_{\mathrm{A}}, w_{\mathrm{B}}$ and $D_{\mathrm{A}}^{*}, D_{\mathrm{B}}^{*}$ are [13]:

$$
D_{\mathrm{A}(\mathrm{B})}^{*}=\left(z \lambda^{2} / 6\right) c_{\mathrm{V}} w_{\mathrm{A}(\mathrm{B})} f_{\mathrm{A}(\mathrm{B})},
$$

where $z$ is the coordination number of the lattice, $\lambda$ is the jump distance, $f_{\mathrm{A}}$ and $f_{\mathrm{B}}$ are the tracer correlation factors for species A and B respectively. It has been shown by Moleko and Allnatt [15] that exact relations (sum-rules) exist between the phenomenological coefficients of the random alloy model in addition to the Onsager reciprocity relations. In a binary random alloy with a cubic lattice these sum-rules are expressed as:

$$
L_{\mathrm{AA}(\mathrm{BB})}+L_{\mathrm{AB}} w_{\mathrm{A}(\mathrm{B})} / w_{\mathrm{B}(\mathrm{A})}=\left(z \lambda^{2} / 6 \Omega k T\right) c_{\mathrm{V}} c_{\mathrm{A}(\mathrm{B})} w_{\mathrm{A}(\mathrm{B})} \text {. }
$$

Then, for the lower limit in Eq. 17, we have that:

$$
D^{(\mathrm{SS})} / \varphi_{\mathrm{V}}=\left(z \lambda^{2} / 6\right)\left(c_{\mathrm{A}}+c_{\mathrm{B}}\right) w_{\mathrm{A}} w_{\mathrm{B}} /\left(c_{\mathrm{A}} w_{\mathrm{B}}+c_{\mathrm{B}} w_{\mathrm{A}}\right)
$$

whilst for the upper limit:

$$
D_{\mathrm{V}}=\left(z \lambda^{2} / 6\right)\left(c_{\mathrm{A}}+c_{\mathrm{B}}\right)^{-1}\left(c_{\mathrm{A}} w_{\mathrm{A}}+c_{\mathrm{B}} w_{\mathrm{B}}\right)\left\{1-\left(6 \Omega k T / z \lambda^{2}\right) L_{\mathrm{AB}}\left(w_{\mathrm{A}}-w_{\mathrm{B}}\right)^{2} / c_{\mathrm{V}}\left(c_{\mathrm{A}} w_{\mathrm{A}}+c_{\mathrm{B}} w_{\mathrm{B}}\right) w_{\mathrm{A}} w_{\mathrm{B}}\right\} .
$$

Unfortunately, the Moleko and Allnatt sum-rules are not enough to express the upper limit in Eq. 17 in terms of $w_{\mathrm{A}}$ and $w_{\mathrm{B}}$. The Manning diffusion kinetics random alloy formalism [13] provides an approximation for the relationships between the phenomenological coefficients and the atomic jump frequencies $w_{\mathrm{A}}$ and $w_{\mathrm{B}}$ in a binary alloy at $c_{\mathrm{V}} \rightarrow 0$. These relationships satisfy the Onsager reciprocity relations as well as the sum-rules discovered much later by Moleko and Allnatt [15]. Since we have already applied the Moleko and Allnatt sum-rules, here, we will write down only one of the relationships, namely:

$$
L_{\mathrm{AB}}=\left(z \lambda^{2} / 3 \Omega k T\right) c_{\mathrm{V}} c_{\mathrm{A}} c_{\mathrm{B}} w_{\mathrm{A}} w_{\mathrm{B}} /\left[H\left(c_{\mathrm{A}}+c_{\mathrm{B}}\right)+2\left(c_{\mathrm{A}} w_{\mathrm{B}}+c_{\mathrm{B}} w_{\mathrm{A}}\right)\right],
$$

where

$$
H=\frac{c_{\mathrm{A}} w_{\mathrm{B}}+c_{\mathrm{B}} w_{\mathrm{A}}}{\left(1-f_{0}\right)\left(c_{\mathrm{A}}+c_{\mathrm{B}}\right)}-w_{\mathrm{A}}-w_{\mathrm{B}}+\left[\left(\frac{c_{\mathrm{A}} w_{\mathrm{B}}+c_{\mathrm{B}} w_{\mathrm{A}}}{\left(1-f_{0}\right)\left(c_{\mathrm{A}}+c_{\mathrm{B}}\right)}-w_{\mathrm{A}}-w_{\mathrm{B}}\right)^{2}+\frac{4 f_{0} w_{\mathrm{A}} w_{\mathrm{B}}}{1-f_{0}}\right]^{\frac{1}{2}} .
$$

Later, a self-consistent theory describing the phenomenological coefficients in a random alloy was presented by Moleko, Allnatt and Allnatt (MAA) [16]. This theory, as was noted first by Belova and Murch [17], gives relationships between the phenomenological coefficients and the atomic jump frequencies in a binary alloy at $c_{\mathrm{V}} \rightarrow 0$ that are exactly equivalent to those obtained by Manning [13]. However, the relationships between the phenomenological coefficients and the tracer diffusion coefficients of the atomic components $D_{\mathrm{A}}^{*}$ and $D_{\mathrm{B}}^{*}$ for a binary alloy are different between the Manning [13] and MAA 
[16] formalisms, as shown by the considerable difference of the results for the tracer correlation factors of the atomic components [18]. The tracer correlation factors given by Manning [13] are:

$$
f_{\mathrm{A}(\mathrm{B})}=H /\left(H+2 w_{\mathrm{A}(\mathrm{B})}\right),
$$

whilst the MAA [16] result can be written as (see also [18]):

$$
f_{\mathrm{A}(\mathrm{B})}=\left[-B_{\mathrm{A}(\mathrm{B})}+\left(B_{\mathrm{A}(\mathrm{B})}^{2}-4 A_{\mathrm{A}(\mathrm{B})} C_{\mathrm{A}(\mathrm{B})}\right)^{\frac{1}{2}}\right] / 2 A_{\mathrm{A}(\mathrm{B})},
$$

where $A_{\mathrm{A}(\mathrm{B})}=H\left(f_{0}\left(c_{\mathrm{A}} w_{\mathrm{A}}+c_{\mathrm{B}} w_{\mathrm{B}}\right) /\left(c_{\mathrm{A}}+c_{\mathrm{B}}\right)+\left(1-f_{0}\right) w_{\mathrm{A}(\mathrm{B})}\right)+2 w_{\mathrm{A}(\mathrm{B})}\left(\left(1-f_{0}\right)\left(c_{\mathrm{A}} w_{\mathrm{B}}+c_{\mathrm{B}} w_{\mathrm{A}}\right) /\left(c_{\mathrm{A}}+c_{\mathrm{B}}\right)+f_{0} w_{\mathrm{B}(\mathrm{A})}\right)$, $B_{\mathrm{A}(\mathrm{B})}=H\left(\left(c_{\mathrm{A}} w_{\mathrm{B}}+c_{\mathrm{B}} w_{\mathrm{A}}\right) /\left(c_{\mathrm{A}}+c_{\mathrm{B}}\right)-f_{0} w_{\mathrm{A}(\mathrm{B})}\right)-2 f_{0} w_{\mathrm{A}} w_{\mathrm{B}}, \quad C_{\mathrm{A}(\mathrm{B})}=-f_{0} H w_{\mathrm{B}(\mathrm{A})}$. High-precision Monte Carlo calculations by Belova and Murch [17,18] showed that both Manning [13] and MAA [16] formalisms for the phenomenological coefficients expressed in term of the atomic jump frequencies in a binary alloy are in excellent agreement with computer simulation results. However, the Manning [13] formalism generally provides only a fair approximation of the tracer correlation factors of the atomic components. In general, the MAA [16] formalism performs much better than Manning's and the agreement with computer simulation results is almost exact [18].

In practice, in order to estimate the range for the controlling parameter of the vacancy motion within a binary alloy nanoshell, it is reasonable to substitute into Eqs. 18 and $20-25$ the average atomic fractions for the compositions of the species $c_{\mathrm{A}} /\left(c_{\mathrm{A}}+c_{\mathrm{B}}\right) \rightarrow x$ and $c_{\mathrm{B}} /\left(c_{\mathrm{A}}+c_{\mathrm{B}}\right) \rightarrow 1-x$, and the average vacancy composition, $c_{\mathrm{V}}^{\mathrm{eq}}$, with respect to the planar surface in the bulk of the binary alloy for $c_{\mathrm{V}}$.

Finally, in this section it should be noted that if for some hollow binary alloy nanosphere we can expect the vacancy thermodynamic factor close to unity $\varphi_{\mathrm{V}} \approx 1$, then the steady-state approximation for vacancies (Eq. 5) should be quite reasonable and, consequently, we can use Eq. 8 coupled with Eq. 17 and the MAA formalism [16] for the tracer correlation factors to estimate a range for the collapse time of a hollow binary alloy nanosphere:

$$
\left(c_{\mathrm{V}}^{\mathrm{eq}} D_{\mathrm{V}}\right)^{-1}<12 \gamma \Omega t_{\mathrm{c}} / k T r_{\mathrm{f}}^{3} \tau_{\mathrm{c}}<\left(c_{\mathrm{V}}^{\mathrm{eq}} D^{(\mathrm{SS})}\right)^{-1} .
$$

\section{Kinetic Monte Carlo simulation}

Let us consider a kinetic Monte Carlo model of a hollow binary alloy nanosphere on a rigid f.c.c. lattice with lattice parameter $a$. We assume that the three nearest neighbour pair-interaction energies are equal $\phi_{\mathrm{AA}}=\phi_{\mathrm{BB}}=\phi_{\mathrm{AB}}=\phi$ but the atomic jump frequencies are strongly different $w_{\mathrm{A}} / w_{\mathrm{B}}=100$. This model has an enthalpy of mixing equal to zero and it is a perfect approximation of the random alloy model. The reduced pair-interaction energies $\phi / k T=-1.5$ provides an equilibrium vacancy composition $c_{\mathrm{V}}^{\mathrm{eq}} \sim 1.24 \times 10^{-4}$ close to the vacancy composition at the melting temperature $T_{\mathrm{m}}$ (for further details see $[11,12,19])$. Furthermore, for such a model, the vacancy thermodynamic factor should be equal to unity $\left(\varphi_{\mathrm{V}}=1\right)$.

The initial hollow nanosphere had an inner radius $r_{\mathrm{i} 0}=7 a$ and external radius $r_{\mathrm{e} 0}=17 a\left(\varepsilon_{0} \approx 0.41\right)$, and consisted of $N_{\mathrm{A}}+N_{\mathrm{B}}=76750$ atoms randomly distributed on the lattice of the nanoshell according to alloy compositions. No initial vacancies were introduced inside the nanoshell. We chose the Metropolis et al. algorithm for its transparency and to provide a clear relationship between KMC steps per atom (KMCSA) $n$ and real time $t$. For this case, the relation evidently is $t=n / \Gamma_{0}$, where $\Gamma_{0}=12 v_{0}, v_{0}=x v_{0 \mathrm{~A}}+(1-x) v_{0 \mathrm{~B}}, v_{0 \mathrm{~A}}$ and $v_{0 \mathrm{~B}}$ are the effective attempt frequencies of $\mathrm{A}$ and $\mathrm{B}$ atoms, respectively. The different jump frequencies $w_{\mathrm{A}}$ and $w_{\mathrm{B}}$ for exchanges with a vacancy in this model are provided by using different attempt frequencies. Furthermore, because $c_{\mathrm{V}}^{\text {eq }}$ is quite small for this model we can neglect the probability to find a divacancy inside of the nanoshell, so that yields $w_{\mathrm{A}}=v_{0 \mathrm{~A}}, w_{\mathrm{B}}=v_{0 \mathrm{~B}}$ and $v_{0}=x w_{\mathrm{A}}+(1-x) w_{\mathrm{B}}$. In all our calculations $v_{0}$ was considered 
as a constant while the atomic fraction $x$ was varied from 0.05 to 0.95 depending on the alloy model. Hence, for such a KMC model the atomic jump frequencies inside a nanoshell in units of $v_{0}$ as the functions of the atomic fraction $x$ at the given ratio $v_{0 \mathrm{~A}} / v_{0 \mathrm{~B}}(=100$ in our simulation) are simply given by $w_{\mathrm{A}} / v_{0}=\left(v_{0 \mathrm{~A}} / v_{0 \mathrm{~B}}\right)\left[\left(v_{0 \mathrm{~A}} / v_{0 \mathrm{~B}}-1\right) x+1\right]^{-1}$ and $w_{\mathrm{B}} / v_{0}=\left[\left(v_{0 \mathrm{~A}} / v_{0 \mathrm{~B}}-1\right) x+1\right]^{-1}$.

Then, in full analogy with Eq. 26 assuming the steady-state approximation for the vacancies (Eq. 5) and taking into account that the average vacancy jump frequency $w_{\mathrm{V}}=x w_{\mathrm{A}}+(1-x) w_{\mathrm{B}}=v_{0}=$ const , the range for the normalized collapse time of the KMC model of the hollow random binary alloy nanosphere is given by:

$$
\left[1-\frac{2 x(1-x)\left(w_{\mathrm{A}}-w_{\mathrm{B}}\right)^{2}}{\left(x w_{\mathrm{A}}+(1-x) w_{\mathrm{B}}\right)\left[H+2\left(x w_{\mathrm{B}}+(1-x) w_{\mathrm{A}}\right)\right]}\right]^{-1}<\frac{t_{\mathrm{c}}\left(x, w_{\mathrm{A}} / w_{\mathrm{B}}\right)}{t_{\mathrm{c}}\left(w_{\mathrm{A}} / w_{\mathrm{B}}=1\right)}<1+\frac{x(1-x)\left(w_{\mathrm{A}}-w_{\mathrm{B}}\right)^{2}}{w_{\mathrm{A}} w_{\mathrm{B}}} .
$$

On the other hand, the normalized collapse time of the KMC model of the hollow random binary alloy nanosphere can be determined directly from the simulation by the relation: $t_{\mathrm{c}}\left(x, w_{\mathrm{A}} / w_{\mathrm{B}}\right) / t_{\mathrm{c}}\left(w_{\mathrm{A}} / w_{\mathrm{B}}=1\right)=n_{\mathrm{c}}\left(x, w_{\mathrm{A}} / w_{\mathrm{B}}\right) / n_{\mathrm{c}}\left(w_{\mathrm{A}} / w_{\mathrm{B}}=1\right)$, where $n_{\mathrm{c}}\left(x, w_{\mathrm{A}} / w_{\mathrm{B}}\right)$ is the number of KMC steps per atom before collapse of the hollow nanosphere with given $x$ and ratio $w_{\mathrm{A}} / w_{\mathrm{B}}$. In Fig. 1(a), the normalized collapse time as a function of the atomic fraction $x$ according to the data of KMC simulation is shown by points, together with solid curves predicted by the lower and upper limits of Eq. 27. Fig. 1(a) clearly demonstrates that the collapse time of the KMC model of the hollow random binary alloy nanosphere is more than could be expected due to the correlation factor of the vacancies for selfdiffusion. It is because the diffusion of species A proceeds faster, and then A segregates near the inner surface. The resulting composition gradient of component A reduces the vacancy flux and hence generates a tendency to suppress the shrinking process [9]. At the same time, the collapse time of the KMC model of the hollow random binary alloy nanosphere is much smaller than could be expected at a steady-state approximation for the atomic components (see Fig. 1(a)). Thus, the results of the KMC simulation demonstrate that the steady-state condition for the atomic components is not reachable during the shrinkage of the hollow random binary alloy nanosphere with the atomic jump frequency ratio $w_{\mathrm{A}} / w_{\mathrm{B}}=100$ at any composition. In other words, the collapse time of the KMC model of the hollow random binary alloy nanosphere is much less than the

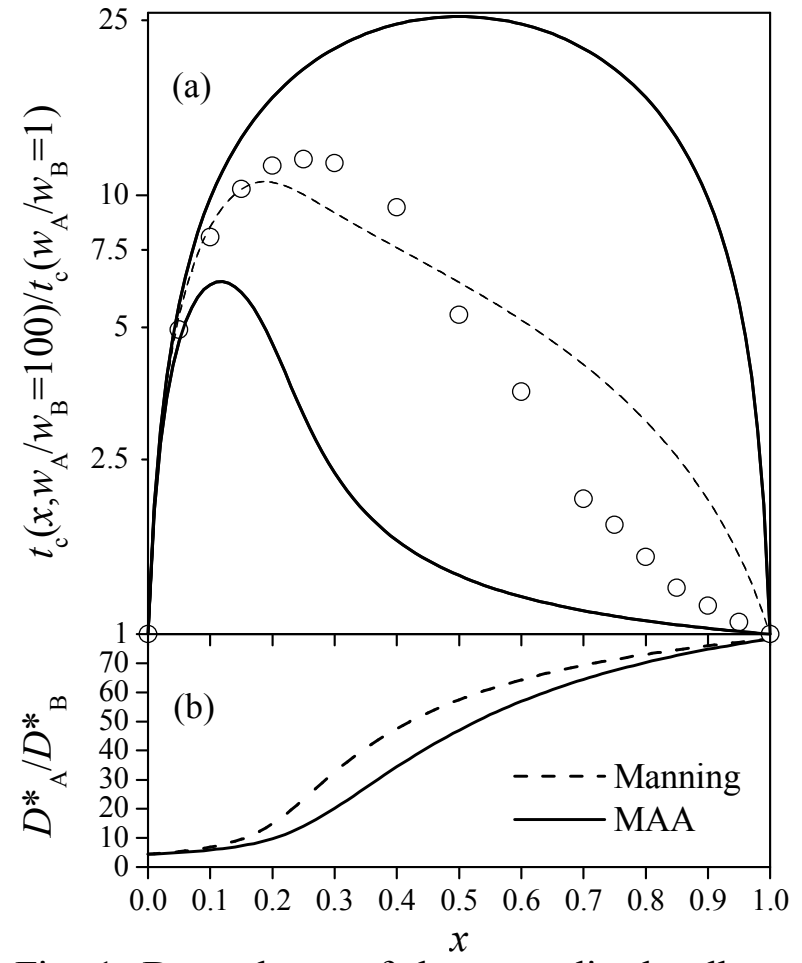

Fig. 1. Dependence of the normalized collapse time (a) and the tracer diffusion coefficient ratio (b) on the atomic fraction of $\mathrm{A}$ for the hollow random binary alloy nanospheres $\left(\varepsilon_{0} \approx 0.41\right)$ with $w_{\mathrm{A}} / w_{\mathrm{B}}=100$. (a) - The points are results of the KMC simulation; the upper and lower solid lines correspond to the upper and lower limits of the Eq. 27, respectively; the dashed line corresponds to the controlling parameter of the vacancy motion within a binary alloy nanoshell with the ratio of the atomic fluxes given by Eq. 28. (b) Dashed and solid lines correspond to Manning [13] and MAA [16] formalisms, respectively. time required for achieving the steady-state condition for the atomic components. Fig. 1(b) shows that the tracer diffusion coefficient ratio $D_{\mathrm{A}}^{*} / D_{\mathrm{B}}^{*}$ of the components of the random binary alloys as a function of the atomic fraction $x$ at the given ratio $w_{\mathrm{A}} / w_{\mathrm{B}}=100$ for Manning [13] and MAA [16] formalisms, respectively. It can be seen from Fig. 1 (a) and (b) that the steady-state condition for the atomic 
components is not reachable during the shrinkage of the hollow random binary alloy nanosphere at any composition at least if the tracer diffusion coefficient ratio $D_{\mathrm{A}}^{*} / D_{\mathrm{B}}^{*}$ is about one - two orders of magnitude.

Finally, it is important to point out one of our observations, which is purely empirical and has no strong justifiable physical proof. With the aid of Eq. 8 a reasonable approximation of the dependence of the collapse time of the KMC model of the hollow random binary alloy nanosphere on the atomic fraction $x$ at $x \leq 0.5$ can be obtained in the following way. We use in Eq. 12 the geometric mean of the ratios of the atomic fluxes at self-diffusion (Eq. 13) and steady-state (Eq. 15) for the controlling parameter of the vacancy motion within the nanoshell:

$$
\frac{J_{\mathrm{A}}}{J_{\mathrm{B}}}=\left(\frac{J_{\mathrm{A}}^{*}}{J_{\mathrm{B}}^{*}} \frac{J_{\mathrm{A}}^{(\mathrm{SS})}}{J_{\mathrm{B}}^{(\mathrm{SS})}}\right)^{\frac{1}{2}}=\left(\frac{x\left(L_{\mathrm{AA}}+L_{\mathrm{AB}}\right)}{(1-x)\left(L_{\mathrm{BB}}+L_{\mathrm{AB}}\right)}\right)^{\frac{1}{2}}=\frac{x}{1-x}\left(\frac{w_{\mathrm{A}}\left(H+2 w_{\mathrm{B}}\right)}{w_{\mathrm{B}}\left(H+2 w_{\mathrm{A}}\right)}\right)^{\frac{1}{2}} .
$$

The predicted (according to Eq. 8) normalized collapse time for the controlling parameter of the vacancy motion within a binary alloy nanoshell (Eq. 12) with the ratio of the atomic fluxes given by Eq. 28 is shown in Fig. 1(a) by the dashed line.

\section{Summary}

By a theoretical description and kinetic Monte Carlo simulations, it has been demonstrated that for a hollow random binary alloy nanosphere (initially homogeneous) at an approximation neglecting the radial dependence of the vacancy formation free energy, the steady-state condition for the atomic components is not reachable during its shrinkage at any composition at least if the tracer diffusion coefficient ratio $D_{\mathrm{A}}^{*} / D_{\mathrm{B}}^{*}$ is about one - two orders of magnitude. A reasonable approximation of the dependence of the collapse time of the kinetic Monte Carlo model of the hollow random binary alloy nanosphere on the atomic fraction $x$ of the faster diffusion species at $x \leq 0.5$ can be obtained by using the geometric mean of the ratios of the atomic fluxes at selfdiffusion and steady-state in the theoretical description of the vacancy motion within the nanoshell. At $x>0.5$ the ratio of the atomic fluxes approaches the self-diffusion ratio as $x$ increases.

\section{Acknowledgments}

This research was supported by the Australian Research Council. One of us (E.V.L.) wishes to thank the University of Newcastle for the award of a Research Fellowship.

\section{References}

[1] H.C. Zeng: J. Mater.Chem. Vol. 16 (2006), p. 649.

[2] H.J. Fan, U. Gösele, M. Zacharias: Small Vol. 3 (2007), p. 1660.

[3] Y Y. Sun, B. Mayers, Y. Xia: Adv. Mater. Vol. 15 (2003), p. 641.

[4] Y. Yin, R.M. Rioux, C.K. Erdonmez, et al.: Science Vol. 304 (2004), p. 711.

[5] R. Nakamura, J.-G. Lee, H. Mori, H. Nakajima: Phil. Mag. Vol. 88 (2008), p. 257.

[6] R. Tenne: Angew. Chem. Vol. 115 (2003), p. 5280.

[7] J. Goldberger, R. Fan, P. D. Yang: Acc. Chem. Res. Vol. 39 (2006), p. 239.

[8] K.N. Tu and U. Gösele: Appl. Phys. Lett. Vol. 86 (2005), p. 093111.

[9] A.M. Gusak, T.V. Zaporozhets, K.N. Tu and U. Gösele: Phil. Mag. Vol. 85 (2005), p. 4445.

[10] A.V. Evteev, E.V. Levchenko, I.V. Belova, G.E. Murch: Phil. Mag., in press.

[11] A.V. Evteev, E.V. Levchenko, I.V. Belova, G.E. Murch: Phil. Mag. Vol. 87 (2007), p. 3787.

[12] A.V. Evteev, E.V. Levchenko, I.V. Belova, G.E. Murch: Def. Diff. Forum Vol. 277 (2008), p. 21.

[13] J.R. Manning: Diffusion Kinetics for Atoms in Crystals (Van Nostrand Reinhold, Princeton 1968).

[14] A.R. Allnatt, A.B. Lidiard: Atomic Transport in Solids (Cambridge University Press, Cambridge 1993).

[15] L.K. Moleko, A.R. Allnatt: Phil. Mag. A Vol. 58 (1988), p. 677.

[16] L.K. Moleko, A.R. Allnatt, E.L. Allnatt: Phil. Mag. A Vol. 59 (1989), p. 141.

[17] I.V. Belova, G.E. Murch: Phil. Mag. A Vol. 80 (2000), p. 599.

[18] I.V. Belova, G.E. Murch: Phil. Mag. A Vol 80 (2000), p. 1469.

[19] A.V. Evteev, E.V. Levchenko, I.V. Belova, G.E. Murch: Def. Diff. Forum Vol. 266 (2007), p. 39. 
Diffusion in Materials - DIMAT2008

doi:10.4028/www.scientific.net/DDF.289-292

Composition Effect on Shrinkage of Hollow Binary Alloy Nanospheres

doi:10.4028/www.scientific.net/DDF.289-292.665

\section{References}

[1] H.C. Zeng: J. Mater.Chem. Vol. 16 (2006), p. 649.

doi:10.1039/b511296f

[2] H.J. Fan, U. Gösele, M. Zacharias: Small Vol. 3 (2007), p. 1660.

doi:10.1002/smll.200700382

PMid:17890644

[3] Y Y. Sun, B. Mayers, Y. Xia: Adv. Mater. Vol. 15 (2003), p. 641.

doi:10.1002/adma.200301639

[4] Y. Yin, R.M. Rioux, C.K. Erdonmez, et al.: Science Vol. 304 (2004), p. 711.

doi:10.1126/science. 1096566

PMid:15118156

[5] R. Nakamura, J.-G. Lee, H. Mori, H. Nakajima: Phil. Mag. Vol. 88 (2008), p. 257.

doi:10.1080/14786430701819203

[6] R. Tenne: Angew. Chem. Vol. 115 (2003), p. 5280.

doi:10.1002/ange.200301651

[7] J. Goldberger, R. Fan, P. D. Yang: Acc. Chem. Res. Vol. 39 (2006), p. 239. doi:10.1021/ar040274h

PMid:16618091

[8] K.N. Tu and U. Gösele: Appl. Phys. Lett. Vol. 86 (2005), p. 093111.

doi:10.1063/1.1873044

[9] A.M. Gusak, T.V. Zaporozhets, K.N. Tu and U. Gösele: Phil. Mag. Vol. 85 (2005), p. 4445.

doi:10.1080/14786430500311741

[10] A.V. Evteev, E.V. Levchenko, I.V. Belova, G.E. Murch: Phil. Mag., in press.

[11] A.V. Evteev, E.V. Levchenko, I.V. Belova, G.E. Murch: Phil. Mag. Vol. 87 (2007), p. 3787.

doi:10.1080/14786430601103005

[12] A.V. Evteev, E.V. Levchenko, I.V. Belova, G.E. Murch: Def. Diff. Forum Vol. 277 (2008), p. 21. 
doi:10.4028/www.scientific.net/DDF.277.21

[13] J.R. Manning: Diffusion Kinetics for Atoms in Crystals (Van Nostrand Reinhold, Princeton 1968).

[14] A.R. Allnatt, A.B. Lidiard: Atomic Transport in Solids (Cambridge University Press, Cambridge 1993).

[15] L.K. Moleko, A.R. Allnatt: Phil. Mag. A Vol. 58 (1988), p. 677.

doi:10.1080/01418618808209945

[16] L.K. Moleko, A.R. Allnatt, E.L. Allnatt: Phil. Mag. A Vol. 59 (1989), p. 141.

doi:10.1080/01418618908220335

[17] I.V. Belova, G.E. Murch: Phil. Mag. A Vol. 80 (2000), p. 599.

doi:10.1080/01418610008212070

[18] I.V. Belova, G.E. Murch: Phil. Mag. A Vol 80 (2000), p. 1469.

doi:10.1080/01418610008212131

[19] A.V. Evteev, E.V. Levchenko, I.V. Belova, G.E. Murch: Def. Diff. Forum Vol. 266 (2007), p. 39.

doi:10.4028/www.scientific.net/DDF.266.39 